Proceedings

\title{
Investigation on the Influence of Solvents on MWCNT-PDMS Nanocomposite Pressure Sensitive Films ${ }^{+}$
}

\author{
Rajarajan Ramalingame *, Pritha Chandraker and Olfa Kanoun \\ Technische Universität Chemnitz, Reichenhainer Straße 70, 09126 Chemnitz, Germany; \\ pritha.chandraker@s2014.tu-chemnitz.de (P.C.); olfa.kanoun@etit.tu-chemnitz.de (O.K.) \\ * Correspondence: rajarajan.ramalingame@etit.tu-chemnitz.de; Tel.: +49-176-729-71362 \\ + Presented at the Eurosensors 2017 Conference, Paris, France, 3-6 September 2017.
}

Published: 16 August 2017

\begin{abstract}
Nanocomposites based on Multi-Walled Carbon Nanotubes (MWCNT)Polydimethylsiloxane (PDMS) polymer achieve pressure sensors sensitive even at very low pressure less than $5 \mathrm{~N}$. For film homogeneity and sensitivity, fabrication procedure and especially the dispersion quality are decisive. Because of high viscosity of PDMS, a solvent is necessary. Solvents exhibit themselves different dispersion quality and in turn different piezoresistive response of the films under pressure. In this paper, the influence of solvents in fabricating the nanocomposite is investigated considering dispersion quality and stability. The best dispersion stability was achieved with isopropanol and the nanocomposite show better results considering sensitivity and hysteresis behavior under pressure.
\end{abstract}

Keywords: carbon nanotube; MWCNT; PDMS; pressure sensor; dispersion quality; solvents; nanocomposites

\section{Introduction}

In the process of fabricating a polymer nanocomposite based pressure sensor, effective dispersion of MWCNT in the polymer matrix is of great importance as it influences the percolation threshold and therefore the mechanical sensitivity of the sensor and its measurement range. The viscosity of PDMS base polymer is 5.1 Pa-sec [1], exceeding the nominal limit of ultra-sonication for direct dispersion of MWCNTs. A silicone thinner could reduce the viscosity of PDMS but will also reduce the self-life and sensitivity of the nanocomposite senor. Hence, to decrease the viscosity and establish a stable dispersion of MWCNTs in PDMS solvents could be used as solvents can be easily evaporated from the nanocomposite. Dispersion of MWCNTs in solvents is well studied in literature and some strong organic solvents like chloroform, tetrahydrofuran (THF), dimethylformamide (DMF), n-methyl-2pyrrolidone (NMP) has shown good and stable dispersion quality for MWCNTs [2].

In this paper, in addition to the above-mentioned solvents, isopropanol is also being investigated for its dispersion property, as it is comparatively less hazardous. Solvents could exhibit different dispersion qualities with MWCNTS but miscibility of the solvent to PDMS is more important for the nanocomposite. An immiscible solvent could retain the MWCNTs within the solvent rather than distributing them into the polymer matrix. Solvents like DMF and NMP exhibit lack of compatibility with PDMS as they show vigorous reaction upon mixing with PDMS, forming dense white gel like structure. Hence, DMF and NMP are not considered for this investigation in-spite of their excellent dispersion properties [2]. Isopropanol on the other hand shows miscibility with PDMS up to $36 \mathrm{~h}$ and could be considered for short term stable nanocomposites. 


\section{Materials and Methods}

To study the dispersing quality of the solvents, an optimum concentration of $0.1 \mathrm{mg} / \mathrm{mL}$ of pristine MWCNT form Sigma Aldrich with relative purity $>95 \%$, outer diameter 6-9 $\mathrm{nm}$ and length $>1 \mu \mathrm{m}$ was chosen. Any higher concentration could adversely degrade the dispersion quality and stability. $0.5 \mathrm{mg}$ of MWCNTs was sonicated in $5 \mathrm{~g}$ of solvent at $15 \%$ amplitude for $30 \mathrm{~min}$ using a horn sonicator (Sonoplus HD 7300, horn diameter $3 \mathrm{~mm}$, Bandelin electronic GmbH, Berlin, Germany). The dispersions are then diluted in the corresponding solvents in the ratio 1:50 to obtain the necessary transparency for UV-vis analysis. The dispersions are stored in standard conditions for long-term analysis.

For a cost-effective pressure sensor, less concentration of MWCNTs like $0.8 \mathrm{wt} . \%, 0.6 \mathrm{wt} . \%$ and 0.4 wt.\% in PDMS were chosen. A measurable DC resistance approx. $40 \mathrm{M} \Omega$ was obtained for a concentration of $0.6 \mathrm{wt} . \%$. Hence, this concentration was mixed with the solvents and dispersed using horn sonicator for $30 \mathrm{~min}$ at $15 \%$ amplitude. Then the dispersions were stirred in $5 \mathrm{~g}$ of PDMS using the magnetic stirrer CAT-M26 for $60 \mathrm{~min}$ at $1600 \mathrm{rpm}$ at $70^{\circ} \mathrm{C}$. After cooling, curing agent was mixed gently to the nanocomposites in the ratio 10:1. Later, the nanocomposites were placed in the degassing chamber for $30 \mathrm{~min}$ to aid solvent evaporation and eliminate trapped air bubbles. The nanocomposites are then casted PTFE molds of $20 \mathrm{~mm}$ diameter and $0.5 \mathrm{~mm}$ height and cured in the oven for $10 \mathrm{~h}$ at $60^{\circ} \mathrm{C}$.

\section{Results and Discussion}

\subsection{Visual Inspection}

At a sonication amplitude of $15 \%$ for $30 \mathrm{~min}$ a good dispersion of MWCNTs in solvents were obtained. After sonication, no visible agglomerations were seen with homogenously dispersed MWCNTs. The dispersion stability degrades over time across all solvents, with toluene to be the most unstable up to $10 \mathrm{~h}$ and isopropanol to most stable over 6 days. Figure 1 shows few agglomerations in isopropanol dispersion after 10 days. Figure 2 shows the degree of dispersion stability in all the solvents after 10 days and it can be observed that, toluene exhibits very poor dispersion stability compared to other solvents. The stability in other solvents cannot be visually determined and hence a UV-vis spectroscopy analysis is required.

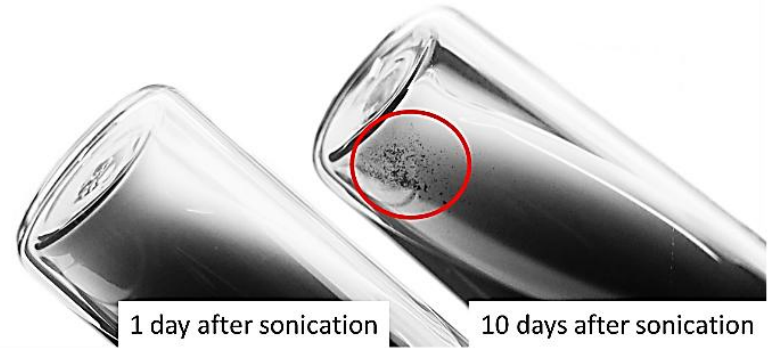

(a)

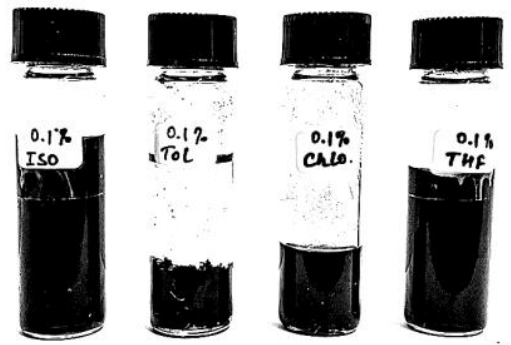

(b)

Figure 1. Visual inspection of the dispersion quality: (a) Formation of agglomerations after few days of dispersion; (b) Settling down of MWCNTs in Toluene compared to other solvents.

\subsection{UV Visible Spectroscopy Analysis}

UV-vis analysis was performed using Perkin Elmer-Lambda 900 on all the dispersions with wavelength ranging from $350 \mathrm{~nm}$ to $1000 \mathrm{~nm}$. Figure 2 shows the characteristic absorbance curves for solvents without MWCNTs (light blue curve) and for dispersions from the day of sonication until 8 days. It can be seen from Figure $2 \mathrm{~d}$, as the MWCNT agglomerations increases the absorbance curve moves towards the solvent's absorbance curve, indications of poor stability. Based on this analysis the degree of dispersion stability over days can be represented as shown in Figure 3 and is in the order: isopropanol $>\mathrm{THF}>$ chloroform $>$ toluene. 


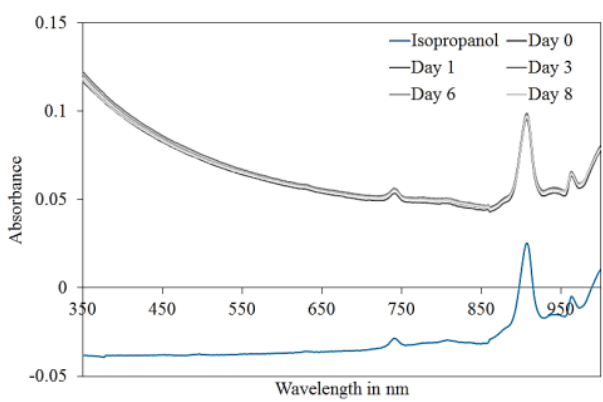

(a)

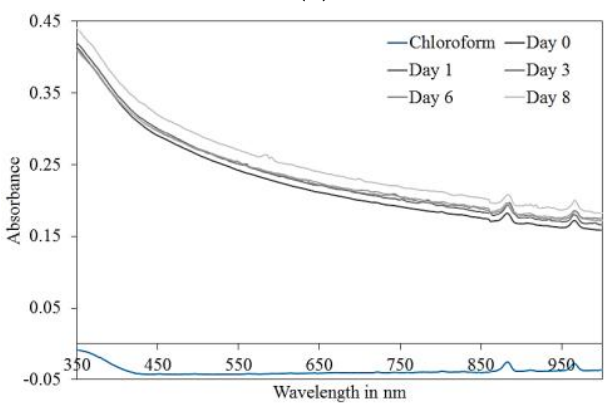

(c)

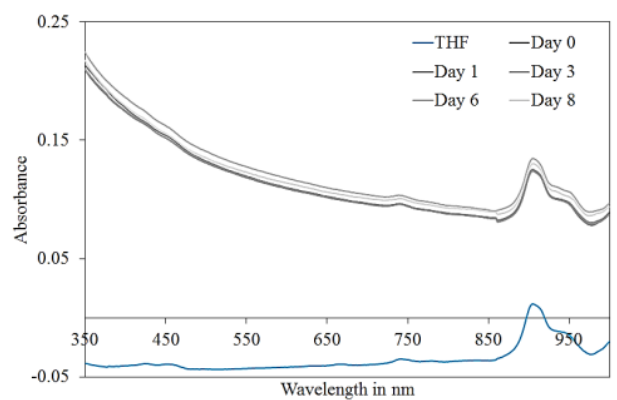

(b)

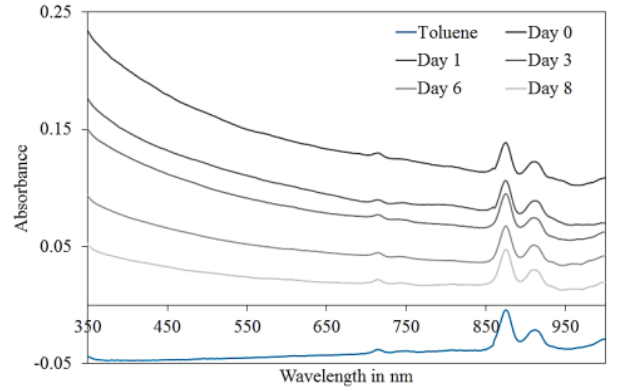

(d)

Figure 2. Absorbance curves obtained with UV-visible spectroscopy up to 8 days from dispersion: (a) Isopropanol; (b) THF; (c) Chloroform; (d) Toluene.

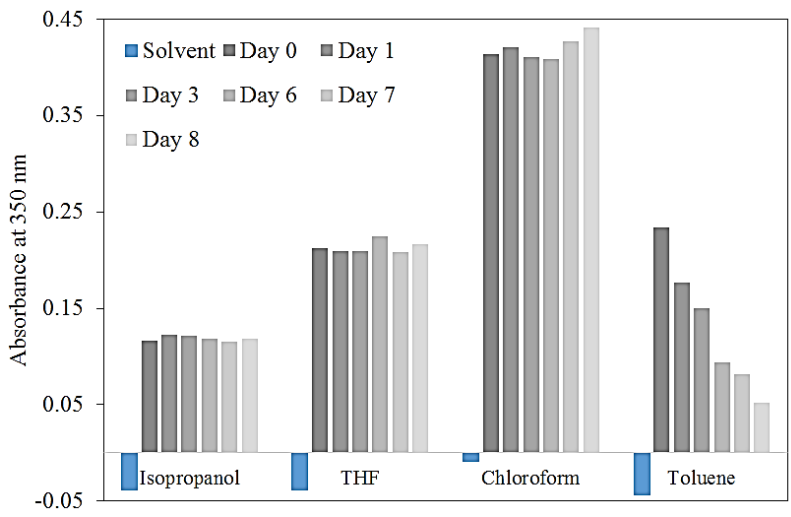

Figure 3. Stability analysis-absorbance obtained with UV-visible spectroscopy at $350 \mathrm{~nm}$ wavelength for all the solvents and dispersions up to 8 days.

\subsection{Performance Under Pressure}

The initial resistance of the fabricated sensors ranges from $12 \mathrm{M} \Omega$ to $43 \mathrm{M} \Omega$ depending on the solvents, small for good dispersion and large for bad dispersion as few conductive paths are established with the same concentration. All sensors were subjected to a maximum load of $10 \mathrm{~N}$ and was applied using a custom-built pressure test bench at a rate of $0.1 \mathrm{~N} / \mathrm{s}$. Under pressure, the dispersed MWCNTs in the polymer matrix are brought closer forming more conduction paths as the tunneling gap between MWCNTs are greatly reduced. This behavior can be clearly seen in Figure 4 as a negative relative change in resistance. Figure 4 shows the pressure response for all the sensors and it can be observed that a maximum relative change in resistance of up to $80 \%$ at $5 \mathrm{~N}$ is obtained for all the samples which is factor of the concentration of MWCNTs. It can also be inferred that, nanocomposites with good dispersion quality exhibits less hysteresis and better sensitivity. Hysteresis and pressure sensing range of the sensors is directly proportional to the quality and stability of the dispersion. Isopropanol and THF exhibit similar hysteresis compared to chloroform 
and toluene. Toluene on the other hand, due to the poor dispersion quality MWCNTs might be settled down in the sensor film and exhibits a switch response under applied load.

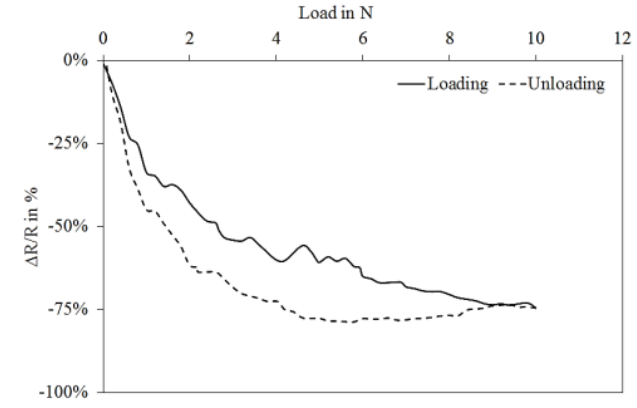

(a)

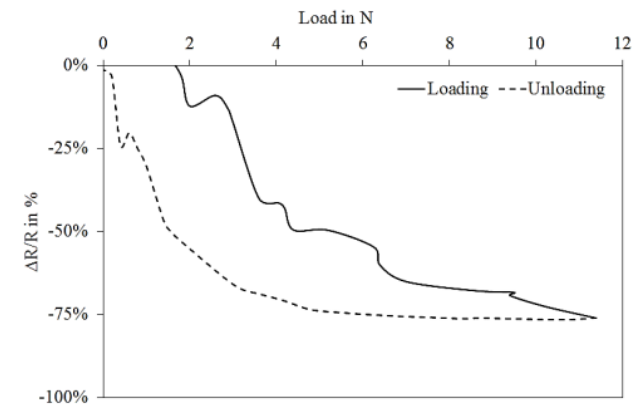

(c)

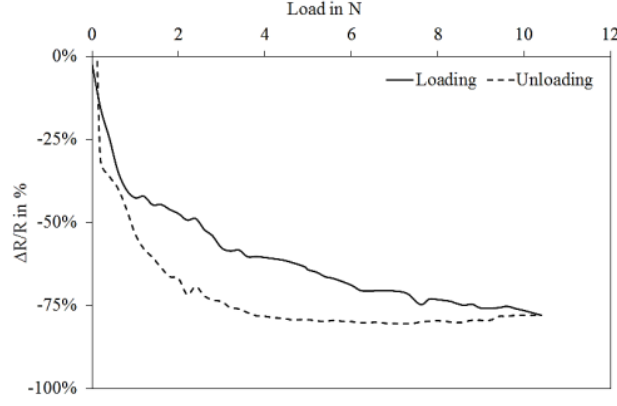

(b)

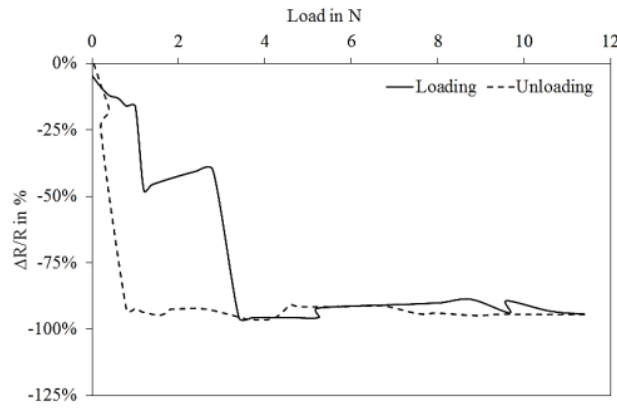

(d)

Figure 4. Influence of solvent on pressure sensing property of the nanocomposite sensors: (a) Isopropanol; (b) THF; (c) Chloroform; (d) Toluene.

\section{Conclusions}

In this work the influence of solvents in MWCNT-PDMS based pressure sensors have been investigated. Toluene based sensors exhibited poor dispersion quality and stability, which resulted in poor pressure sensing behavior. Isopropanol and THF bears nearly the same dispersion qualities and good sensitivity to pressure, isopropanol based sensors exhibits less hysteresis compared to that of THF based sensors. Chloroform based sensors are in-between the above two with moderate dispersion stability and good sensitivity to pressure but large hysteresis.

Acknowledgments: The research work is being funded by "Europäischer Sozialfonds" in the form of scholarship for the Ph.D. The Professorship of Electrical Measurements and Sensor Technology, Chemnitz University of Technology under the guidance of Olfa Kanoun, provides the research facility as well as the cost for conference and open access publication.

Conflicts of Interest: The founding sponsor "Europäischer Sozialfonds", had no role in the design of the study; in the collection, analyses, or interpretation of data; in the writing of the manuscript, and in the decision to publish the results.

\section{References}

1. Hong, J.; Lee, J.; Hong, C.K.; Shim, S.E. Effect of dispersion state of carbon nanotube on the thermal conductivity of polydimethyl siloxane composites. Curr. Appl. Phys. 2010, 10, 359-363.

2. Liu, C.-X.; Choi, J.-W. Improved Dispersion of Carbon Nanotubes in Polymers at High Concentrations. Nanomaterials 2012, 2, 329-347.

3. Bauhofer, W.; Kovacs, J.Z. A review and analysis of electrical percolation in carbon nanotube polymer composites. Compos. Sci. Technol. 2009, 69, 1486-1498.

(C) 2017 by the authors. Licensee MDPI, Basel, Switzerland. This article is an open access article distributed under the terms and conditions of the Creative Commons Attribution (CC BY) license (http://creativecommons.org/licenses/by/4.0/). 